\title{
HOLOMORPHIC MAPPINGS OF POLYDISCS INTO COMPACT COMPLEX MANIFOLDS
}

\author{
K. KODAIRA
}

In this paper we prove an inequality in the manner of the Nevanlinna theory expressing certain properties of holomorphic mappings of $n$-dimensional polydiscs into compact complex manifolds of the same dimension and discuss some of its applications.

1. Let $W$ be a compact complex manifold of dimension $n$. For a point $w$ in $W$, we denote a local coordinate of $w$ by $\left(w^{1}, w^{2}, \cdots, w^{n}\right)$. Take a complex line bundle $L$ over $W$. By a theorem of de Rham, the Chern class $c(L)$ of $L$ can be regarded as a $d$-cohomology class of $d$-closed 2 -forms on $W$. We say that a real $(1,1)$-form

$$
\gamma=i \sum_{\alpha, \beta=1}^{n} g_{\alpha \beta}(w) d w^{\alpha} \wedge d \bar{w}^{\beta}, \quad i=\sqrt{-1},
$$

on $W$ is positive semidefinite (or positive definite) if the Hermitian matrix $\left(g_{\alpha \beta}(w)\right)_{\alpha, \beta=1, \cdots, n}$ is positive semidefinite (or positive definite) at every point $w \in W$. Denote the canonical bundle of $W$ by $K$. In this section we assume the existence of a complex line bundle $L$ over $W$ together with a positive integer $m$ satisfying the following condition: The Chern class $c(L)$ contains a positive semidefinite $d$-closed real $(1,1)$-form and

$$
\operatorname{dim} H^{0}\left(W, \mathcal{O}\left(K^{m} \otimes L^{-1}\right)\right)>0,
$$

where $\mathcal{O}\left(K^{m} \otimes L^{-1}\right)$ denotes the sheaf over $W$ of germs of holomorphic sections of $K^{m} \otimes L^{-1}$.

Cover $W$ by a finite number of small neighborhoods $U_{j}, j=1,2, \ldots$, and fix a local coordinate: $w \rightarrow\left(w_{j}^{1}, \cdots, w_{j}^{n}\right)$ on each $U_{j}$. Take a 1-cocycle $\left\{l_{j k}\right\}$ determining the line bundle $L$ composed of nonvanishing holomorphic functions $l_{j k}=l_{j k}(w)$ defined, respectively, on $U_{j} \cap U_{k}$. We then find a 0-cochain $\left\{a_{j}\right\}$ composed of $C^{\infty}$-differentiable functions $a_{j}=a_{j}(w)>0$ defined, respectively, on $U_{j}$ satisfying

$$
a_{j}(w)^{m}=\left|l_{j k}(w)\right|^{2} a_{k}(w)^{m}, \quad \text { on } U_{j} \cap U_{k},
$$

such that 


$$
\gamma=i \sum_{\alpha, \beta=1}^{n} g_{j \alpha \beta}(w) d w_{j}^{\alpha} \wedge d \bar{w}_{j}^{\beta}=i \partial \bar{\partial} \log a_{j}(w)
$$

is positive semidefinite. Note that the $d$-closed real $(1,1)$-form $m \gamma$ belongs to the Chern class $c(L)$. We choose a holomorphic section

$$
\varphi \in H^{0}\left(W, \mathcal{O}\left(K^{m} \otimes L^{-1}\right)\right), \quad \varphi \neq 0,
$$

and denote by $\varphi_{j}(w)$ the fibre coordinate of $\varphi(w)$ over $U_{j}$. It is clear that

$$
v=a_{j}(w)\left|\varphi_{j}(w)\right|^{2 / m}(i / 2)^{n} d w_{j}^{1} \wedge d \bar{w}_{j}^{1} \wedge \cdots \wedge d w_{j}^{n} \wedge d \bar{w}_{j}^{n}
$$

is a volume element, i.e., a real continuous $2 n$-form which is nonnegative everywhere on $W$. Fix a point $p^{0} \in W$ such that $\varphi\left(p^{0}\right) \neq 0$, and assume that $p^{0} \in U_{1}$. We normalize the volume element $v$ by the condition:

$$
a_{1}\left(p^{0}\right)\left|\varphi_{1}\left(p^{0}\right)\right|^{2 / m}=1 \text {. }
$$

Let $\mathbf{C}^{n}$ denote the space of $n$ complex variables, define $|z|=\max _{\lambda}\left|z_{\lambda}\right|$ for $z=\left(z_{1}, \cdots, z_{\lambda}, \cdots, z_{n}\right) \in \mathbf{C}^{n}$, and denote by $\Delta_{r}$ a polydisc of radius $r$ :

$$
\Delta_{r}=\left\{z \in \mathbf{C}^{n}|| z \mid<r\right\} .
$$

Take a polydisc $\Delta_{R} \subseteq \mathbf{C}^{n}$, consider a holomorphic mapping $f$ of $\Delta_{R}$ into $W$, and assume that the Jacobian of $f$ does not vanish at the origin $0 \in \Delta_{R}$ and that

$$
f(0)=p^{0}
$$

For simplicity we write

$$
d V(z)=(i / 2)^{n} d z_{1} \wedge d \bar{z}_{1} \wedge \cdots \wedge d z_{n} \wedge d \bar{z}_{n},
$$

and let $f^{*}(v)$ denote the volume element on $\Delta_{R}$ induced from $v$ by the mapping $f$. Then we have

$$
f^{*}(v)=\xi(z) d V(z), \quad \xi(z)=a_{j}(f(z))\left|\varphi_{j}(f(z))\right|^{2 / m}\left|J_{j}(z)\right|^{2},
$$

where

$$
J_{j}(z)=\operatorname{det}\left(\partial w_{i j}^{\alpha} / \partial z_{\lambda}\right)_{\alpha, \lambda=1, \cdots, n}, \quad\left(w_{j}^{1}, \cdots, w_{j}^{n}\right)=f(z) .
$$

By hypothesis the Jacobian $J_{j}(z)$ of $f$ does not vanish identically, and therefore the equation $\xi(z)=0$ defines a proper analytic subset of $\Delta_{R}$. Hence, by applying a suitable linear transformation to $\mathbf{C}^{n}$ if necessary, we may assume that, for any fixed values of $z_{1}, \cdots, z_{\lambda-1}, z_{\lambda+1}, \cdots, z_{n}$, the function $\xi\left(z_{1}, \cdots, z_{\lambda}, \cdots, z_{n}\right)$ of $z_{\lambda}$ does not vanish identically and that 


$$
J_{1}(0)=1 \text {. }
$$

Set

$$
\begin{aligned}
\sigma_{\lambda}=(i / 2)^{n-1} d z_{1} & \wedge d \bar{z}_{1} \wedge \cdots \wedge d \bar{z}_{\lambda-1} \wedge d z_{\lambda+1} \wedge \cdots \wedge d \bar{z}_{n}, \\
\sigma & =\sum_{\lambda=1}^{n} \sigma_{\lambda}, \\
\left|\partial f(z) / \partial z_{2}\right|^{2} & =\sum_{\alpha, \beta=1}^{n} g_{j \alpha \beta}(f(z))\left(\partial w_{j}^{\alpha} / \partial z_{\lambda}\right)\left(\partial \bar{w}_{j}^{\beta} / \partial \bar{z}_{\lambda}\right),
\end{aligned}
$$

where $\left(w_{j}^{1}, \cdots, w_{j}^{n}\right)=f(z)$. Moreover, setting $z_{\lambda}=r_{\lambda} e^{i \theta_{\lambda}}$, we introduce polar coordinates $\left(r_{\lambda}, \theta_{\lambda}\right)$ and let

$$
d S(z)=\sum_{\lambda=1}^{n} r_{\lambda} d \theta_{\lambda} \wedge \sigma_{\lambda} .
$$

We denote the bundary of the polydisc $\Delta_{r}$ by $\partial \Delta_{r}$.

Now we define functions $M(r), A(r)$ and $N(r)$ of $r, 0<r<R$, as follows:

$$
\begin{aligned}
& M(r)=r^{-1} \int_{\partial \Delta_{r}} \log \xi(z) d S(z), \\
& A(r)=4 \int_{\Delta_{r}} \sum_{\lambda=1}^{n}\left|\partial f(z) / \partial z_{\lambda}\right|^{2} d V(z), \\
& N(r)=4 \pi m^{-1} \int_{\left(f^{*} \varphi\right) \cap \Delta_{r}} \sigma+4 \pi \int_{(J) \cap \Delta_{r}} \sigma,
\end{aligned}
$$

where $\left(f^{*} \varphi\right)$ and $(J)$ denote, respectively, the divisors of the holomorphic functions $\varphi_{j}(f(z))$ and $J_{j}(z)$.

Theorem 1. We have the inequality:

$$
\int_{0}^{r} A(t) t^{-1} d t+\int_{0}^{r} N(t) t^{-1} d t \leq M(r) .
$$

Proof. Let

$$
\mu(z)=\log \xi(z) .
$$

The set $\Gamma=\{z \mid \xi(z)=0\}$ is a proper analytic subset of $\Delta_{R}$, and $\mu(z)$ is $C^{\infty}$ differentiable outside $\Gamma$. For brevity we write

$$
z=\left(z_{1}, \zeta\right), \quad \zeta=\left(z_{2}, \cdots, z_{n}\right)
$$

We set 


$$
\mu_{1}(r, \zeta)=\int_{0}^{2 \pi} \mu\left(r e^{i \theta}, \zeta\right) d \theta
$$

Lemma. $\mu_{1}(r, \zeta)$ is a continuous function of $(r, \zeta), 0<r<R,|\zeta|<R$, and is a piecewise smooth function of $r, 0<r<R$, when $\zeta$ is fixed.

To prove this lemma, take a point $\zeta^{0},\left|\zeta^{0}\right|<R$, and a real number $r^{0}, 0<$ $r^{0}<R$, such that $\left(r^{0} e^{i \theta}, \zeta^{0}\right) \notin \Gamma$ for $0 \leq \theta<2 \pi$. Moreover, for each $\zeta,|\zeta|<R$, denote by $\rho_{h}(\zeta), h=1,2,3, \cdots$, the roots of the equation:

$$
\varphi_{j}\left(f\left(z_{1}, \zeta\right)\right) J_{j}\left(z_{1}, \zeta\right)^{m}=0 \text {. }
$$

Then for a small positive number $\varepsilon$ we have, for $\left|z_{1}\right|<r^{0},\left|\zeta-\zeta^{0}\right|<\varepsilon$,

$$
\mu(z)=2 m^{-1} \sum_{h} \log \left|z_{1}-\rho_{h}(\zeta)\right|+\tau(z),
$$

where the summation is extended over all roots $\rho_{h}(\zeta)$ with $\left|\rho_{h}(\zeta)\right|<r^{0}$, and $\tau(z)$ is a $C^{\infty}$-differentiable function of $z$. Using the formula

$$
\int_{0}^{2 \pi} \log \left|r e^{i \theta}-\rho\right| d \theta=2 \pi \max \{\log r, \log |\rho|\},
$$

we hence obtain

$$
\mu_{1}(r, \zeta)=4 \pi m^{-1} \sum_{h} \max \left\{\log r, \log \left|\rho_{h}(\zeta)\right|\right\}+\tau_{1}(r, \zeta),
$$

where $\tau_{1}(r, \zeta)$ is a $C^{\infty}$-differentiable function of $(r, \zeta),|r|<r_{0},\left|\zeta-\zeta^{0}\right|<\varepsilon$. Since the roots $\rho_{h}(\zeta)$, arranged in an appropriate order, are continuous functions of $\zeta,\left|\zeta-\zeta^{0}\right|<\varepsilon$, the formula (6) proves the lemma.

Define

$$
M\left(r_{1}, r_{2}, \cdots, r_{n}\right)=\int \mu\left(z_{1}, z_{2}, \cdots, z_{n}\right) d \theta_{1} d \theta_{2} \cdots d \theta_{n},
$$

where the integral is extended over the domain: $0 \leq \theta_{1}<2 \pi, 0 \leq \theta_{2}<2 \pi$, $\cdots, 0 \leq \theta_{n}<2 \pi$. Since

$$
M\left(r_{1}, r_{2}, \cdots, r_{n}\right)=\int \mu_{1}\left(r_{1}, z_{2}, \cdots, z_{n}\right) d \theta_{2} \cdots d \theta_{n},
$$

we infer from the above lemma that $M\left(r_{1}, r_{2}, \ldots, r_{n}\right)$ is a continuous function of $\left(r_{1}, r_{2}, \cdots, r_{n}\right) \neq(0, \cdots, 0)$, while, by (2), (3) and (4), the function $\mu(z)$ of $z$ is $C^{\infty}$-differentiable in a neighborhood of 0 . Consequently $M\left(r_{1}, \cdots r_{n}\right)$ is a continuous function of $\left(r_{1}, \cdots, r_{n}\right), 0 \leq r_{\lambda}<R$.

Let $\partial_{1}$ denote the exterior differentiation with respect to the variable $z_{1}$. We then have 


$$
i \partial_{1} \bar{\partial}_{1} \mu(z)=i \partial_{1} \bar{\partial}_{1} \log a_{j}(f(z))=\left|\partial f(z) / \partial z_{1}\right|^{2} i d z_{1} \wedge d \bar{z}_{1}
$$

Define

$$
B(r, \zeta)=\int_{\left|z_{1}\right|<r} 2 i \partial_{1} \bar{\partial}_{1} \mu(z)=\int_{\left|z_{1}\right|<r} 2\left|\partial f(z) / \partial z_{1}\right|^{2} i d z_{1} \wedge d \bar{z}_{1} .
$$

Setting $z_{1}=x+i y$, we have

$$
2 i \partial_{1} \bar{\partial}_{1} \mu=d * d \mu, \quad * d \mu=(\partial \mu / \partial x) d y-(\partial \mu / \partial y) d x .
$$

Moreover the function $\mu\left(z_{1}, \zeta\right)$ is $C^{\infty}$-differentiable in $z_{1}$ for $z_{1} \neq \rho_{h}(\zeta)$. Hence, letting

$$
\oint_{\rho} * d \mu(z)=\lim _{\varepsilon \rightarrow 0} \int_{\left|z_{1}-\rho\right|=\varepsilon} * d \mu\left(z_{1}, \zeta\right)
$$

we obtain

$$
B(r, \zeta)=\int_{\left|z_{1}\right|=r} * d \mu(z)-\sum_{|\rho|<r} \oint_{\rho} * d \mu(z)
$$

Note that $\oint_{\rho} * d \mu(z)=0$ for $\rho \neq \rho_{h}(\zeta), h=1,2, \ldots$. We denote by $\nu\left(r, \zeta, f^{*} \varphi\right)$ and $\nu(r, \zeta, J)$, respectively, the number of the roots on the disc $\left|z_{1}\right|<r$ of the equations $\varphi\left(f\left(z_{1}, \zeta\right)\right)=0$ and $J_{j}(z, \zeta)=0$. Since

$$
\mu(z)=\log a_{j}(f(z))+2 m^{-1} \log \left|\varphi_{j}(f(z))\right|+2 \log \left|J_{j}(z)\right|,
$$

we have

$$
\sum_{|\rho|<r} \oint_{\rho} * d \mu(z)=4 \pi m^{-1} \nu\left(r, \zeta, f^{*} \varphi\right)+4 \pi \nu(r, \zeta, J) .
$$

Moreover we see readily that

$$
\int_{\left|z_{1}\right|=r} * d \mu(z)=r \partial \mu_{1}(r, \zeta) / \partial r
$$

Hence, setting

$$
\nu(r, \zeta)=4 \pi m^{-1} \nu\left(r, \zeta, f^{*} \varphi\right)+4 \pi \nu(r, \zeta, J)
$$

we obtain

$$
B(r, \zeta)+\nu(r, \zeta)=r \partial \mu_{1}(r, \zeta) / \partial r
$$


and therefore

$$
\int_{s}^{r} B(t, \zeta) t^{-1} d t+\int_{s}^{r} \nu(t, \zeta) t^{-1} d t=\mu_{1}(r, \zeta)-\mu_{1}(s, \zeta)
$$

This proves the inequality

$$
\mu_{1}\left(r, z_{2}, \cdots, z_{n}\right) \geq \mu_{1}\left(s, z_{2}, \cdots, z_{n}\right), \quad \text { for } r>s>0 .
$$

It follows that

$$
M\left(r, r_{2}, \cdots, r_{n}\right) \geq M\left(s, r_{2}, \cdots, r_{n}\right), \quad \text { for } r>s .
$$

Thus we infer that $M\left(r_{1}, \cdots, r_{\lambda}, \cdots, r_{n}\right)$ is a monotone nondecreasing function of each variable $r_{\lambda}$. Since, by (2), (3) and (4), $\xi(0)$ is equal to 1 , we get

$$
M\left(r_{1}, r_{2}, \cdots, r_{n}\right) \geq 0 .
$$

Define

$$
\begin{aligned}
A(t, u) & =\int_{|\zeta| \leq u} B(t, \zeta) d V(\zeta), \\
N(t, u) & =\int_{|\zeta| \leq u} \nu(t, \zeta) d V(\zeta), \\
M_{1}(t, u) & =\int_{|\zeta| \leq u} \mu_{1}(t, \zeta) d V(\zeta),
\end{aligned}
$$

where

$$
d V(\zeta)=\sigma_{1}=(i / 2)^{n-1} d z_{2} \wedge d \bar{z}_{2} \wedge \cdots \wedge d z_{n} \wedge d \bar{z}_{n} .
$$

Since $i d z_{\lambda} \wedge d \bar{z}_{\lambda}=2 r_{\lambda} d r_{\lambda} d \theta_{\lambda}$, we have

$$
M_{1}(r, u)=\int_{0}^{u} M\left(r, r_{2}, r_{3}, \cdots, r_{n}\right) r_{2} d r_{2} r_{3} d r_{3} \cdots r_{n} d r_{n},
$$

where the integral is extended over the domain: $0 \leq r_{\lambda} \leq u, \lambda=2,3, \cdots, n$. Hence, using (8), we obtain from (7) the inequality

$$
\int_{0}^{r} A(t, u) t^{-1} d t+\int_{0}^{r} N(t, u) t^{-1} d t \leq M_{1}(r, u) .
$$

Set 


$$
\begin{aligned}
& M_{\lambda}(r)=\int_{0}^{r} M\left(t_{2}, \cdots, t_{\lambda}, r, t_{\lambda+1}, \cdots, t_{n}\right) t_{2} d t_{2} \cdots t_{n} d t_{n}, \\
& A_{\lambda}(r)=4 \int_{\Delta_{r}}\left|\partial f(z) / \partial z_{\lambda}\right|^{2} d V(z), \\
& N_{\lambda}(r)=4 \pi m^{-1} \int_{\left(f^{*}\right) \cap \Delta_{r}} \sigma_{\lambda}+4 \pi \int_{(J) \cap \Delta_{r}} \sigma_{\lambda} .
\end{aligned}
$$

Since $M_{1}(r)=M_{1}(r, r), A_{1}(t)=A(t, t) \leq A(t, u)$ and $N_{1}(t)=N(t, t) \leq N(t, u)$ for $t \leq u$, we derive from (9) the inequality

$$
\int_{0}^{r} A_{1}(t) t^{-1} d t+\int_{0}^{r} N_{1}(t) t^{-1} d t \leq M_{1}(t)
$$

We infer in the same manner that

$$
\int_{0}^{r} A_{\lambda}(t) t^{-1} d t+\int_{0}^{r} N_{\lambda}(t) t^{-1} d t \leq M_{\lambda}(t) .
$$

Since

$$
r M(r)=\int_{\partial \Delta_{r}} \mu(z) d S(z)=\sum_{\lambda=1}^{n} \int_{|z|=\left|z_{\lambda}\right|=r} \mu(z) r_{\lambda} d \theta_{\lambda} \wedge d \sigma_{\lambda}
$$

we have

$$
M(r)=\sum_{\lambda=1}^{n} M_{\lambda}(r)
$$

while it is obvious that

$$
A(t)=\sum_{\lambda=1}^{n} A_{\lambda}(t), \quad N(t)=\sum_{\lambda=1}^{n} N_{\lambda}(t) .
$$

Hence the inequality (5) follows from (10). q.e.d.

For a positive number $\beta$, we define

$$
\Omega_{\beta}(r)=\int_{\partial \Delta_{r}} \xi(z)^{\beta} d S(z),
$$

and set

$$
S(r)=\int_{\partial \Delta_{r}} d S(z)=2 n \pi^{n} r^{2 n-1}
$$


Theorem 2. We have the inequality

$$
\int_{0}^{r} A(t) t^{-1} d t+\int_{0}^{r} N(t) t^{-1} d t \leq \beta^{-1} r^{-1} S(r) \log \left(\Omega_{\beta}(r) / S(r)\right) .
$$

Proof. Since $\log x$ is a concave function of $x, x>0$, we have

$$
\begin{aligned}
r M(r) & =\int_{\partial \Delta_{r}} \log \xi(z) d S(z)=\beta^{-1} \int_{\partial \Delta_{r}} \log \xi(z)^{\beta} d S(z) \\
& \leq \beta^{-1} S(r) \log \left(S(r)^{-1} \int_{\partial \Delta_{r}} \xi(z)^{\beta} d S(z)\right)
\end{aligned}
$$

which together with (5) gives the inequality (11). q.e.d.

We have assumed so far that the system of coordinates $\left(z_{1}, \cdots, z_{\lambda}, \cdots, z_{n}\right)$ is general in the sense that, for each $\lambda$ and any fixed values of $z_{1}, \cdots, z_{\lambda-1}$, $z_{\lambda+1}, \cdots, z_{n}$, the function $\xi\left(z_{1}, \cdots, z_{\lambda}, \cdots, z_{n}\right)$ of $z_{\lambda}$ does not vanish identically. However, this assumption is irrelevant to the inequality (11). The inequality (11) holds for any system of coordinates $\left(z_{1}, \cdots, z_{n}\right)$ satisfying the conditions (3) and (4). To prove this, suppose that the coordinates $\left(z_{1}, \cdots, z_{n}\right)$ are obtained from a fixed system of coordinates $\left(z_{1}^{(0)}, \cdots, z_{n}^{(0)}\right)$ by means of a linear transformation $u=\left(u_{\lambda \nu}\right)$ with $\operatorname{det}\left(u_{\lambda \nu}\right)=1$ :

$$
z_{\lambda}=\sum_{\nu=1}^{n} u_{\lambda \nu} z_{\nu}^{(0)}
$$

There exists an everywhere dense subset $G$ of the special linear group $\operatorname{SL}(n, \mathbf{C})$ such that, for every $u \in G$, the corresponding system of coordinates $\left(z_{1}, \cdots, z_{n}\right)$ is general and, consequently, the inequality (11) holds. For our purpose it suffices, therefore, to verify that each term of (11) depends continuously on $u$. It is obvious that $\int_{0}^{r} A(t) t^{-1} d t$ and $\Omega_{\beta}(r)$ are continuous in $u$. Denoting the positive part of $\log x$ by $\log ^{+} x$, we have

$$
\int_{0}^{r} N(t) t^{-1} d t=4 \pi m^{-1} \int_{\left(f^{*} \varphi\right)+m(J)} \log ^{+}(r /|z|) \sigma,
$$

which shows that $\int_{0}^{r} N(t) t^{-1} d t$ depends continuously on $u$. q.e.d.

Note that

$$
\int_{\Delta_{r}} \xi(z)^{\beta} d V(z)=\int_{0}^{r} \Omega_{\beta}(t) d t
$$

Since $A(t)$ and $N(t)$ are nonnegative, the inequality (11) implies that 


$$
\Omega_{\beta}(r) \geq S(r)
$$

Combining this with (12), we get

$$
\int_{\Delta_{r}} \xi(z)^{\beta} d V(z) \geq \pi^{n} r^{2 n}
$$

In particular, setting $\beta=1$, we obtain

$$
\int_{\Delta_{r}} f^{*}(v) \geq \pi^{n} r^{2 n} .
$$

2. A holomorphic mapping is said to be totally degenerate if its Jacobian vanishes identically. Let $v_{0}$ be a volume element which is positive everywhere on $W$. Then, for any holomorphic mapping $f$ of $\Delta_{r}$ into $W$, the quotient $\int_{\Delta_{r}} f^{*}\left(v_{0}\right) / \int_{W} v_{0}$ may be regarded as a mean degree of the mapping $f: \Delta_{r} \rightarrow W$. Define

$$
\operatorname{deg}\left(f \mid \Delta_{r}\right)=\int_{\Delta_{r}} f^{*}\left(v_{0}\right) / \int_{W} v_{0}
$$

and further set

$$
P_{m}=\operatorname{dim} H^{0}\left(W, \mathcal{O}\left(K^{m}\right)\right), \quad \text { for } \quad m_{\AA}^{\top}=1,2,3, \ldots .
$$

Theorem 3. Let $W$ be a compact complex manifold of dimension $n$. If there exists a holomorphic mapping $f$ of $\mathbf{C}^{n}$ into $W$ which is not totally degenerate, and if

$$
\liminf _{r \rightarrow+\infty} r^{-2 n} \operatorname{deg}\left(f \mid \Delta_{r}\right)=0
$$

then all the plurigenera $P_{m}$ of $W$ vanish.

Proof. Suppose that one of the plurigenera, say $P_{m}$, is positive. Then, letting $L$ be a trivial bundle, we have the inequality (1). Hence, by (15), we obtain

$$
\int_{\Delta_{r}} f^{*}(v) \geq \pi^{n} r^{2 n},
$$

which contradicts (16), since the quotient $v / v_{0}$ is bounded on $W$. q.e.d.

By a surface we shall mean a compact complex manifold of dimension 2 . A surface $W$ is said to be regular if the first Betti number $b_{1}(W)$ of $W$ vanishes. $A$ regular surface $W$ is rational if and only if all the plurigenera $P_{m}$ of $W$ vanish (see [9, Theorem 54]). 
Theorem 4. If a regular surface $W$ contains $\mathbf{C}^{2}$ as its open subset, then $W$ is a rational surface.

Proof. Let $W$ be a regular surface containing $\mathbf{C}^{2}$ and let $f: \mathbf{C}^{2} \subseteq W$ denote the inclusion map. It is obvious that $\operatorname{deg}\left(f \mid \Delta_{r}\right)<1$ for each polydisc $\Delta_{r} \subset \mathbf{C}^{2}$. Thus by Theorem 3 all the plurigenera $P_{m}$ of $W$ vanish, and hence $W$ is a rational surface. q.e.d.

Letting $U$ be a non-empty open subset of a compact complex manifold $W$, we call $W$ a compactification of $U$ if the complement $W-U$ of $U$ is an analytic subset of $W$. F. Hirzebruch mentioned in his list [6] of problems the classification of all compactifications of $\mathbf{C}^{n}$. Concerning this problem, A. Van de Ven [13] pointed out that all the known examples of compactifications of $\mathbf{C}^{2}$ are rational surfaces.

Theorem 5. Every compactification of $\mathbf{C}^{2}$ is a rational surface.

Proof. Let $W$ be a compactification of $\mathbf{C}^{2}$. It is then obvious that $b_{1}(W)=$ $b_{1}\left(\mathbf{C}^{2}\right)=0$. Hence, by Theorem $4, W$ is a rational surface. q.e.d.

The condition $\mathbf{C}^{2} \subseteq W$ is much weaker than that $W$ is a compactification of $\mathbf{C}^{2}$. In fact, there exists an infinite sequence of mutually disjoint open subsets $U_{1}, U_{2}, U_{3}, \ldots$ of $\mathbf{C}^{2}$ each of which is biholomorphically isomorphic to $\mathbf{C}^{2}$ (see $\S 4$ below). Thus, if $\mathbf{C}^{2} \subseteq W$, then $U_{1} \subseteq \mathbf{C}^{2} \subseteq W$, and the existence of $U_{1} \subseteq W$ together with the vanishing of $b_{1}(W)$ already implies the rationality of $W$.

3. Letting $W$ be a projective algebraic manifold of dimension $n$, we call $W$ an algebraic manifold of general type if

$$
\limsup _{m \rightarrow+\infty} m^{-n} \operatorname{dim} H^{0}\left(W, \mathcal{O}\left(K^{m}\right)\right)>0
$$

where $K$ denotes the canonical bundle of $W$. Recently Iitaka [7] introduced the concept of canonical dimension. The condition (17) is equivalent to saying that the canonical dimension of $W$ coincides with the dimension $n$ of $W$. In this section we apply Theorem 1 to algebraic manifolds of general type and derive a recent result of Griffiths [5].

Let $W$ be an algebraic manifold of general type of dimension $n, X$ a general hyperplane section of $W$, and $L=[X]$ the complex line bundle over $W$ determined by the divisor $X$. Then, letting $K_{X}$ denote the restriction of $K$ to $X$, we have the exact sequence:

$$
0 \rightarrow H^{0}\left(W, \mathcal{O}\left(K^{m} \otimes L^{-1}\right)\right) \rightarrow H^{0}\left(W, \mathcal{O}\left(K^{m}\right)\right) \rightarrow H^{0}\left(X, \mathcal{O}\left(K_{X}^{m}\right)\right) \rightarrow \cdots,
$$

while $\operatorname{dim} H^{0}\left(X, \mathcal{O}\left(K_{X}^{m}\right)\right)$ is a function of $m$ of order $O\left(m^{n-1}\right)$. Hence, by (17), $\operatorname{dim} H^{0}\left(X, \mathcal{O}\left(K^{m} \otimes L^{-1}\right)\right)$ is positive for a large integer $m$, and thus we have the inequality (1). Obviously we may assume that the real $(1,1)$-form

$$
i \sum g_{j_{\alpha \beta}}(w) d w_{j}^{\alpha} \wedge d \bar{w}_{j}^{\beta}=i \partial \partial \bar{l} \log a_{j}(w)
$$

is positive definite. Therefore, setting 


$$
g_{j}(w)=\operatorname{det}\left(g_{j \alpha \beta}(w)\right),
$$

we find a positive constant $c$ such that

$$
a_{j}(w)\left|\varphi_{j}(w)\right|^{2 / m} \leq c^{n} g_{j}(w), \quad \text { for } w \in W .
$$

Now consider a holomorphic mapping $f: \Delta_{R} \rightarrow W$ satisfying the conditions (3) and (4), and set

$$
\Omega(r)=\Omega_{1 / n}(r), \quad T(r)=\int_{\Delta_{r}} \xi(z)^{1 / n} d V(z) .
$$

Since

$$
g_{j}(f(w))\left|J_{j}(z)\right|^{2} \leq \prod_{\lambda=1}^{n}\left|\partial f(z) / \partial z_{\lambda}\right|^{2}
$$

we have, in consequence of (18),

$$
\xi(z) \leq c^{n} \prod_{\lambda=1}^{n}\left|\partial f(z) / \partial z_{\lambda}\right|^{2}, \quad \xi(z)^{1 / n} \leq n^{-1} c \sum_{\lambda=1}^{n}\left|\partial f(z) / \partial z_{\lambda}\right|^{2},
$$

from which follows

$$
T(r) \leq(4 n)^{-1} c A(r)
$$

Combining this with (11) we obtain

$$
\int_{0}^{r} T(t) t^{-1} d t \leq(4 r)^{-1} c S(r) \log (\Omega(r) / S(r)) .
$$

Set

$$
Q(r)=\int_{0}^{r} T(t) t^{-1} d t, \quad \Psi(r)=2 n \pi^{-n} r^{-2 n} Q(r),
$$

and note that, by (14), $T(r) \geq \pi^{n} r^{2 n}, Q(r) \geq(2 n)^{-1} \pi^{n} r^{2 n}$ and $\Psi(r) \geq 1$. The inequality (19) implies that

$$
r \leq r_{0}, \quad r_{0}=r_{0}(c, n),
$$

where $r_{0}(c, n)$ is a constant depending only on $c$ and $n$ (see Nevanlinna [11, p. 235]). In fact, if $\Omega(r) \leq r^{2} Q(r)^{4}$, then the inequality (19) yields

$$
r^{2} \Psi(r) \leq n^{2} c(4 \log \Psi(r)+(6 n+3) \log r+3 n \log \pi) .
$$


Since $\Psi(r) \geq 1$ and $e \log x \leq x$ for $x>0$, this proves that

$$
r \leq r_{1}=\max \left\{1, n^{2} c e^{-1}(6 n+7)+3 n \log \pi\right\} .
$$

Therefore, if $r>r_{1}$, then (19) implies that $\Omega(r)>r^{2} Q(r)^{4}$. It follows that either $\Omega(r)>T(r)^{2}$ or $T(r)>r Q(r)^{2}$. If $\Omega(r)>T(r)^{2}$, then

$$
d r=\Omega(r)^{-1} d T(r)<T(r)^{-2} d T(r) .
$$

If $T(r)>r Q(r)^{2}$, then

$$
d r=T(r)^{-1} r d Q(r)<Q(r)^{-2} d Q(r) .
$$

Hence we get

$$
\begin{aligned}
r-r_{1} & =\int_{r_{1}}^{r} d t<-\int_{r_{1}}^{r} d\left(T(t)^{-1}+Q(t)^{-1}\right) \\
& <T\left(r_{1}\right)^{-1}+Q\left(r_{1}\right)^{-1}<(2 n+1) \pi^{-n},
\end{aligned}
$$

which proves that

$$
r \leq r_{0}, \quad r_{0}=r_{1}+(2 n+1) \pi^{-n} .
$$

Thus we obtain the following

Theorem 6. Let $W$ be an algebraic manifold of general type, and $p^{0} a$ point on $W$ such that $\varphi\left(p^{0}\right) \neq 0$ for an element $\varphi \in H^{0}\left(W, \mathcal{O}\left(K^{m} \otimes L^{-1}\right)\right)$. Then there exists a constant $r_{0}$ with the following properties: For any holomorphic mapping $f: \Delta_{R} \rightarrow W$ with $f(0)=p^{0}$ and $J_{1}(0)=1$, the inequality $R \leq r_{0}$ holds, where $J_{1}(0)$ denotes the Jacobian of $f$ at the origin 0 .

This theorem has been proved by Griffiths [5] under the assumption that the canonical system $|K|$ is ample. We remark that his proof also applies to the case in which $|K|$ is not assumed to be ample, and establishes the above Theorem 6 (see Kobayashi and Ochiai [8, Addendum]).

4. Bieberbach [2] constructed an example of a biholomorphic mapping $f$ of $\mathbf{C}^{2}$ onto a proper open subset $U$ of $\mathbf{C}^{2}$. His construction is as follows. Let $\eta: z \rightarrow \eta z$ be a biholomorphic automorphism of $\mathbf{C}^{2}$ of which the origin 0 is a fixed point: $\eta 0=0$. Obviously $\eta$ induces a linear transformation of the tangent space $T_{0}\left(\mathbf{C}^{2}\right)\left(\cong \mathbf{C}^{2}\right)$ of $\mathbf{C}^{2}$ at 0 . Let $\lambda$ and $\mu$ denote the eigenvalues of this linear transformation, and assume that $|\lambda| \leq|\mu|<1$. Then there exists a biholomorphic mapping $f_{0}: z \rightarrow f_{0}(z)$ of a neighborhood $N$ of 0 into $\mathbf{C}^{2}$ with $f_{0}(0)=0$ such that $g=f_{0}^{-1} \eta f_{0}$ takes the normal form

$$
g: z=\left(z_{1}, z_{2}\right) \rightarrow g z=\left(\lambda z_{1}+\beta z_{2}^{p}, \mu z_{2}\right),
$$

where $p$ is a positive integer and $\beta$ is a constant which vanishes unless $\lambda=\mu^{p}$ (see Lattès [10], Sternberg [12]). Obviously $g$ is a contraction in the sense that 


$$
\lim _{m \rightarrow+\infty} g^{m} z=0, \quad \text { for } z \in \mathbf{C}^{2}
$$

For every positive integer $m$, we have

$$
f_{0}(z)=\eta^{-m} f_{0}\left(g^{m} z\right), \quad \text { for } z \in N,
$$

provided that $g N \subset N$. Since $\eta^{-m} f_{0} g^{m}$ is defined on $g^{-m} N$ and $\cup_{m} g^{-m} N=\mathbf{C}^{2}$, it follows from (20) that $f_{0}$ can be continued analytically to a biholomorphic mapping $f$ of $\mathbf{C}^{2}$ onto an open subset $U$ of $\mathbf{C}^{2}$ (see Sternberg [12, p. 816]). For every integer $m$ we have

$$
f(z)=\eta^{-m} f\left(g^{m} z\right), \quad \text { for } z \in \mathbf{C}^{2} .
$$

It follows that

$$
U=\left\{z \mid \lim _{m \rightarrow+\infty} \eta^{m} z=0\right\} .
$$

Now we specify $\eta$ to be the automorphism

$$
\eta: z=\left(z_{1}, z_{2}\right) \rightarrow \eta z=\left(z_{2}, \lambda^{2} z_{1}+\left(\lambda^{2}-1\right)\left(\sin z_{2}-z_{2}\right)\right),
$$

where $\lambda$ is a constant with $0<|\lambda|<1$. Note that the normal form of this $\eta$ is

$$
g: z=\left(z_{1}, z_{2}\right) \rightarrow g z=\left(\lambda z_{1},-\lambda z_{2}\right) .
$$

We define a translation

$$
\tau: z=\left(z_{1}, z_{2}\right) \rightarrow\left(z_{1}+2 \pi, z_{2}+2 \pi\right) .
$$

Then $\eta$ and $\tau$ are commutative: $\eta \tau=\tau \eta$, and therefore, for each integer $k$, $\tau^{k} 0=(2 k \pi, 2 k \pi)$ is a fixed point of $\eta$ and

$$
\tau^{k} U=\left\{z \mid \lim _{m \rightarrow+\infty} \eta^{m} z=\tau^{k} 0\right\}
$$

It follows that $\tau^{k} U$ and $\tau^{j} U$ are disjoint for $k \neq j$. Thus we obtain an infinite sequence of mutually disjoint open subsets $\tau^{k} U, k=0, \pm 1, \pm 2, \cdots$, each of which is biholomorphically isomorphic to $\mathbf{C}^{2}$.

Letting $\{\tau\}$ denote the infinite cyclic group generated by $\tau$, we have

$$
\mathbf{C}^{2} /\{\tau)=\mathbf{C}^{*} \times \mathbf{C} \text {. }
$$

Clearly we may regard $U=\bigcup_{k} \tau^{k} U /\{\tau\}$ as an open subset of $\mathbf{C}^{*} \times \mathbf{C}$. Thus we see the existenc of a biholomorphic mapping: $\mathbf{C}^{2} \rightarrow \mathbf{C}^{*} \times \mathbf{C}$. Combining this with Theorem 4 , we infer that if a regular surface $W$ contains $\mathbf{C}^{*} \times \mathbf{C}$ as its open subset, then $W$ is a rational surface. This result can be verified also in the same manner as in the proof of Theorem 4. In fact, if $\mathbf{C}^{*} \times \mathbf{C} \subset W$, then 
$f:\left(z_{1}, z_{2}\right) \rightarrow\left(\exp z_{1}, z_{2}\right)$ is a holomorphic mapping of $\mathbf{C}^{2}$ into $W$ with $\operatorname{deg}\left(f \mid \Delta_{r}\right)$ $=O(r)$. Thus by Theorem 3 all the plurigenera of $W$ vanish, and hence $W$ is a rational surface.

\section{References}

[1] L. V. Ahlfors, Geometrie der Riemannschen Flächen, C. R. Congrès Internat. Math. Oslo, 1936, 239-248.

[2] L. Bieberbach, Beispiel zweier ganzer Funktionen zweier komplexer Variablen welche eine schlicht volumetreue Abbildung des $\mathrm{R}_{4}$ auf einen Teil seiner selbest vermitteln, Preuss. Akad. Wiss. Sitzungsber. 1933, 476-479.

[ 3 ] S. S. Chern, Complex analytic mappings of Riemann surfaces. I, Amer. J. Math. 82 (1960) 323-337.

[4] - The integrated form of the first main theorem for complex analytic mappings in several complex variables, Ann. of Math. 71 (1960) 536-551.

[5] P. A. Griffiths, Holomorphic mappings into canonical algebraic varieties, Ann. of Math. 93 (1971) 439-458.

[6] F. Hirzebruch, Some problems on differentiable and complex manifolds, Ann. of Math. 60 (1954) 213-236.

[ 7 ] S. Iitaka, On D-dimensions of algebraic varieties, Proc. Japan Acad. 46 (1970) 487-489.

[ 8 ] S. Kobayashi \& T. Ochiai, Mappings into compact complex manifolds with negative first Chern classes, J. Math. Soc. Japan 23 (1971) 137-148.

[9] K. Kodaira, On the structure of compact complex analytic surfaces. IV, Amer. J. Math. 90 (1968) 1048-1066.

[10] M. S. Lattès, Sur les formes réduites des transformations ponctuelles à deux variables, C. R. Acad. Sci. Paris 152 (1911) 1566-1569.

[11] R. Nevanlinna, Eindeutige analytische Funktionen, Springer, Berlin, 1936.

[12] S. Sternberg, Local contractions and a theorem of Poincaré, Amer. J. Math. 79 (1957) 809-824.

[13] A. Van de Ven, Analytic compactification of complex homology cells, Math. Ann. 147 (1962) 189-204.

UNIVERSITY OF TOKYO 